\title{
The Effect of Neonatal Nerve Injury on the Expression of Heat Shock Proteins in Developing Rat Motoneurones
}

\author{
BERNADETT KALMÁR, 1,4 GEOFFREY BURNSTOCK, ${ }^{2}$ GERTA VRBOVÁ,3 \\ and LINDA GREENSMITH ${ }^{1}$
}

\begin{abstract}
The expression of the heat shock proteins hsp27 and hsp70 was examined in the spinal cord and sciatic nerves of developing rats. Using immunohistochemistry, we found that hsp27 is present in many motoneurones at birth. With development, the intensity of staining increases, reaching adult levels by 21 days, when all sciatic motoneurones express hsp27. In the sciatic nerve, hsp27 is strongly expressed throughout postnatal development. In contrast, hsp70 immunoreactivity in motoneurones and the sciatic nerve is weak at birth and does not change with development. The expression of heat shock proteins has been shown to increase in cells under conditions of stress, where they have beneficial effects on cell survival. The effect of neonatal nerve injury on hsp27 and hsp70 expression was also examined in this study. Four days after injury, staining for hsp27 increases in motoneurones, whereas hsp70 does not change. However, there is a significant increase in hsp70 staining in glial cells surrounding the injured motor pool, predominantly in astrocytes. Since neonatal nerve injury induces apoptotic motoneurone death, we also studied the co-expression of hsp27 with markers of apoptosis. No hsp27positive motoneurones were found to be apoptotic, as assessed by both TUNEL and caspase-3 immunoreactivity. Therefore, it is possible that the upregulation of hsp27 observed in injured motoneurones may play a role in protecting motoneurones from apoptotic cell death following nerve injury.
\end{abstract}

Key words: astrogliosis; axotomy; caspase-3; development; hsp27; hsp70; TUNEL

\section{INTRODUCTION}

$\mathbf{H}$ EAT SHOCK PROTEINS (hsps) are a large family of proteins that are specifically induced when cells are exposed to various forms of stress (Brown et al., 1989; New et al., 1989; Moreno-Flores et al., 1997; Schlesinger, 1985). They were originally identified and named on the basis of their increased synthesis following exposure to elevated temperatures (Samali and Cotter, 1996; Xia et al., 1998) and have therefore been implicated in pro- tecting cells against stress. Hsps are highly conserved throughout evolution in various organisms ranging from yeast cells to mammalian neurons, and can be induced by many forms of stress, including heat and oxidative injury (Becker and Craig, 1994). They can be divided into five different families according to their molecular weights: 25-30 kDa, $60 \mathrm{kDa}, 70 \mathrm{kDa}, 90 \mathrm{kDa}$, and 105 kDa (Moseley, 1997).

Some hsps are also expressed constitutively (Gernold et al., 1993; Heikkila, 1993; Manzerra and Brown, 1992;

\footnotetext{
${ }^{1}$ Sobell Department of Neurophysiology, Institute of Neurology, London, United Kingdom.

${ }^{2}$ Autonomic Neuroscience Institute, Royal Free Hospital School of Medicine, London, United Kingdom.

${ }^{3}$ Department of Anatomy and Developmental Biology, University College London, London, United Kingdom.

${ }^{4}$ Biorex Research \& Development, Co., Veszprem, Hungary.
} 
Plumier et al., 1997) and in such situations may have several roles. For example, they can act as molecular chaperones during protein synthesis, may be involved in protein transport within the cytoplasm and have been shown to play a role in those processes in which protein folding and refolding are required (Mayer and Brown, 1994). When hsps are upregulated in response to stress they are thought to be able to protect cells by reorganizing damaged proteins and restoring their activities ( $\mathrm{Li}$ et al., 1993; Yenari et al., 1998; Amin et al., 1996). Consistent with this possibility is the observation that mild heat or ischaemic stress, which is sufficient to induce an upregulation in hsp expression, can protect cells during subsequent exposure to more stressful insults. As a consequence, these cells are more likely to survive the second insult than non-conditioned cells (Barbe et al., 1988; Cohen and Downey, 1983; Wagstaff et al., 1999; Sakurai et al., 1998). It is likely that it is not a single class of hsp that is responsible for this protective effect, which is likely to require the expression of a set of several hsps in a coordinated fashion.

In this study, we have examined whether hsps may play a role in the survival of developing motoneurones. It is well established that injury to the peripheral nerve during the early postnatal period is a severe insult that results in the death of a large proportion of motoneurones (Greensmith and Vrbova, 1996; Romanes, 1946). In contrast, in the adult nervous system no motoneurone death takes place following peripheral nerve injury (Lowrie et al., 1982) and most sensory neurones also survive (Coggeshall et al., 1997). Interestingly, in the adult both motor and sensory neurones have been shown to upregulate hsp27 expression following peripheral nerve injury (Costigan et al., 1998; Hopkins et al., 1998).

In order to examine the possible role of hsps in motoneurone survival following neonatal nerve injury, we firstly established the immunoreactivity pattern of hsp27 and hsp70 proteins in motoneurones within the sciatic motor pool, as well as the sciatic nerve during postnatal development. We then tested the effect of peripheral nerve injury in newborn rats on hsp27 and hsp70 immunoreactivity. Since nerve injury is known to induce apoptotic motoneurone death (Bilbao et al., 2000), in order to examine the possible relationship between hsp expression and cell death (Springer et al., 2000; Vanderluit et al., 2000), we also studied in vivo the co-expression of hsps and markers of apoptosis in injured motoneurones.

\section{MATERIALS AND METHODS}

\section{Nerve Injury}

Sprague-Dawley rats of both sexes were used in these experiments (Biological Services, University College
London, U.K.). All experimental animals were cared for in accordance with guidelines issued by the Institute of Neurology and the British Government. On the day of birth (P0), under halothane anaesthesia and sterile conditions, the right sciatic nerve was crushed in the midthigh region, just above the division of the sciatic nerve into the tibial and common peroneal nerves, using a pair of fine Watchmaker's forceps. Following the crush, the nerve was examined to ensure that the epineurial sheath was intact but translucent and the wound was then closed with sutures. Following recovery from anaesthesia, the pups were returned to their mother.

\section{Immunohistochemistry}

Normal and experimental animals were deeply anaesthetized (4\% chloral hydrate, $1 \mathrm{~mL} / 100 \mathrm{~g}$ body weight, i.p.) at various time points (at $1 \mathrm{~h}$ and 1, 2, 4, 7, 14, 21, and 28 days postinjury) and the animals were perfused transcardially with a fixative containing paraformaldehyde (4\% in phosphate-buffered saline [PBS]). At least three animals were examined at each time point in both the operated and control groups. The spinal cords and sciatic nerves from the operated and control hindlimbs (including the site of injury and the proximal and distal nerve portions) were removed, the control and operated sides of the spinal cords identified and the tissues postfixed for $4 \mathrm{~h}$ in the same fixative. Following cryoprotection in sucrose (30\% in PBS), $10-\mu \mathrm{m}$ serial sections from each tissue were cut on a cryostat.

The sections were stained for either hsp27 or hsp70. The sections were incubated for $1 \mathrm{~h}$ in $3 \%$ normal horse serum (NHS; Vector, Burlingame, CA), and then incubated overnight at $4^{\circ} \mathrm{C}$ either in the primary polyclonal antibody to hsp27 (Santa Cruz Biotechnology, Santa Cruz, $\mathrm{CA}$ ) or the monoclonal antibody to hsp70 (Santa Cruz Biotechnology), used at a dilution of 1:100. Negative control sections, in which the primary antibody was omitted, were also prepared. After thorough washing in PBS with 0.1\% Triton-X-100 (Sigma, St. Louis, MO), the sections were incubated for $2 \mathrm{~h}$ at room temperature in biotinylated secondary antibodies (dilution 1:100; Vector), and then incubated for $1 \mathrm{~h}$ in Avidin-Biotin complex (ABC; Vector). They were processed using horseradish peroxidase (HRP) as the chromagen and diamino benzidine (DAB) as the substrate. After drying, the spinal cord sections were counterstained with a Nissl stain (Gallocyanin).

The proportion of sciatic motoneurones immunostained with hsp27 was assessed in control, unoperated animals as well as in those animals in which the sciatic nerve was crushed at birth. In control animals the number of immunostained motoneurones in one ventral horn was counted and in the operated animals, only the ven- 
A

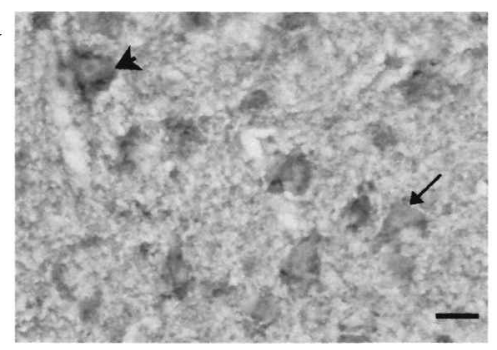

B

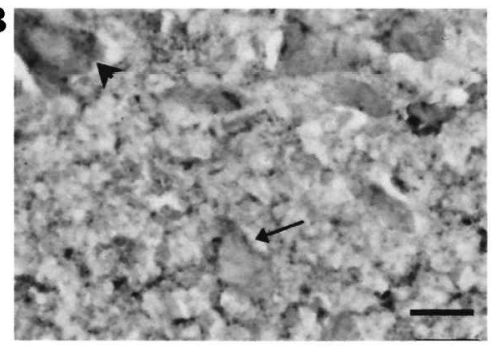

C

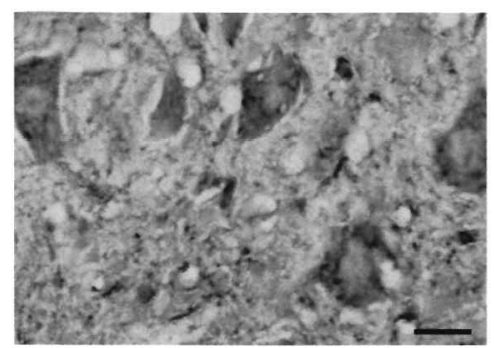

D

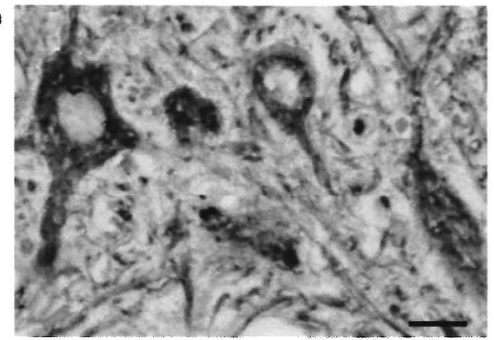

$\mathbf{E}$

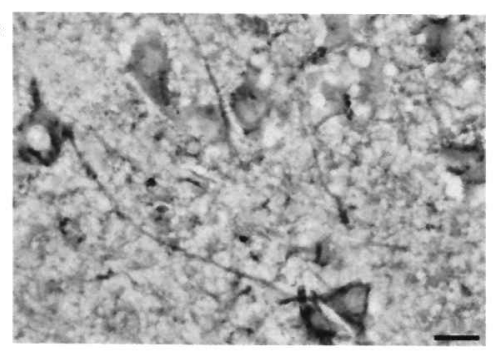

$\mathbf{F}$

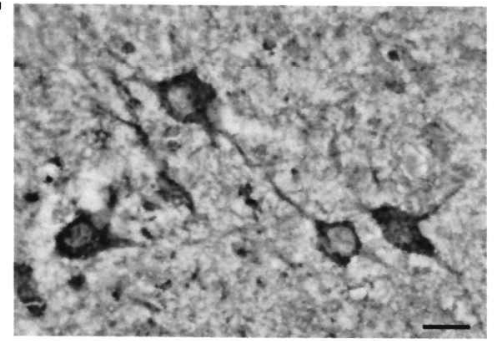

$\mathbf{G}$

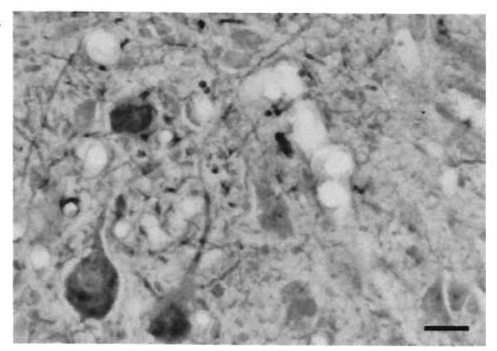

$\mathbf{H}$

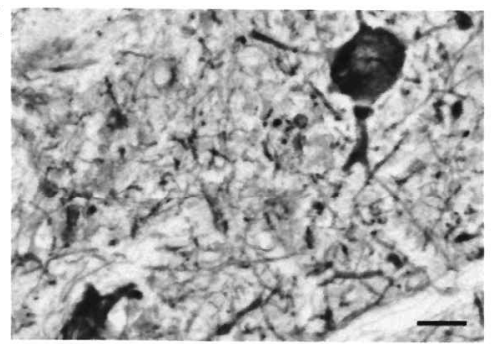

FIG. 1. Developmental pattern of hsp27 expression in spinal cord motoneurones and the effect of neonatal nerve injury. Representative cross-sections of the ventral horn of the lumbar spinal cord stained for hsp27 are shown. The expression of hsp27 was assessed between 1 and 28 days of development and in the adult. The expression pattern of hsp27 immunoreactivity at P4, 7, 14, and 21 is shown in A-D, respectively. At P4 (A) and 7 (B), many motoneurones were hsp27 immunoreactive (large arrowhead), but some motoneurones did not express hsp27 (small arrow, Nissl stained motoneurones). The expression of hsp27 increased until P21 (D), when adult levels were reached and all motoneurones were hsp27 immunoreactive. The effect of neonatal nerve injury on hsp27 expression in motoneurones is illustrated in $\mathbf{E}-\mathbf{H}$ at P4, 7, 14, and 21, respectively. A strong upregulation of hsp27 immunoreactivity in injured motoneurones was observed at P4 (E). This increased hsp27 staining peaks at P7 (F), although it is still elevated at P14 (G). By P21 there is no difference in the expression of hsp27 in injured motoneurones $(\mathrm{H})$ compared to motoneurones on the contralateral control side, although there are fewer surviving motoneurones present in the injured ventral horn. Bar $=20 \mu \mathrm{m}$

tral horn on the side of the nerve injury was assessed. In addition, in both control and operated animals, the total number of sciatic motoneurones stained for either Nissl, or for both Nissl and hsp27 was also established. This assessment of the number of hsp27 immunoreactive motoneurones was carried out at three intervals after injury: (i) 0-4 days, (ii) 7-14 days, and (iii) 21 days to adult. For each animal, five sections were examined and each group consisted of at least three animals. The ratio of immunostained to total number of Nissl-stained motoneurones was calculated. The data is presented as the mean with the standard error of mean (SEM). 


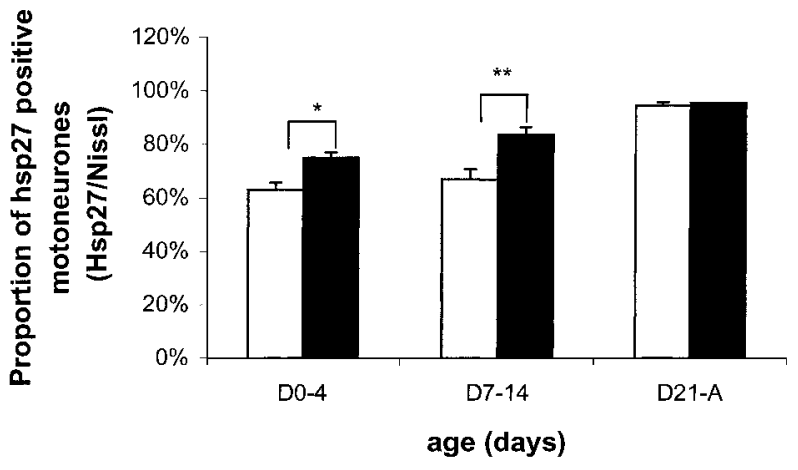

FIG. 2. The proportion of hsp27immunoreactive motoneurones within the sciatic motor pool during postnatal development and following neonatal nerve injury. The bar diagram shows the proportion hsp27 immunoreactive motoneurones within the sciatic motor pool during normal postnatal development (empty bars) and following neonatal nerve injury (filled bars), assessed at three stages of development: P0-P4, P7-P14, and P21 to adult. The number of hsp27-immunoreactive motoneurones in the ventral horn was counted and expressed as a percentage of the total number of Nissl stained motoneurones within the sciatic motor pool. In each group, at least four animals were examined, and the mean number of hsp27 positive motoneurones is expressed as a percentage of the total number of Nissl-stained motoneurones. Error bars show the standard error of mean. Between P0-4 and P7-14, a-significantly greater proportion of sciatic motoneurones are hsp27-immunoreactive following nerve injury compared to uninjured animals at the same stage of development $(* p<0.01 ; * * p<0.02)$.

Co-expression of hsp70 and GFAP. In order to test whether hsp70 expressing cells in the injured spinal cord were astrocytes, we used double labeling for hsp70 and glial fibrillary acidic protein (GFAP), an astroglial marker. The sections were first processed for hsp70 immunoreactivity using the monoclonal mouse antibody. Texas Red Avidin D (Vector, dilution 1:100) was used as fluorescent layer. The same sections were subsequently immunostained for GFAP using a fluorescein-labeled anti-mouse IgG antibody made in horse (Vector; dilution 1:100). Negative control sections were also prepared omitting one of the two antibodies and applying both secondary layers, in order to establish cross-reactivity between antibodies. Sections were examined under a fluorescent microscope, and the presence of hsp70 was assessed using a blue filter for detection of Texas Red, while the presence of GFAP-positive cells was assessed using a green filter for fluorescein.

Hsp70 western blots. Western blot analysis of hsp70 was carried out in spinal cords from unoperated rats $(n=$ $12)$ and operated rats $(n=12)$ at 7 days after nerve crush at birth. Spinal cords were collected in groups of three, so that there were four sets of samples for both the unoperated and operated groups. Each western was run in duplicate and repeated at least twice in order to carry out semiquantitative analysis. The rats were deeply anaesthetised (4\% chloral hydrate, i.p.) and the lumbar spinal cord quickly removed. The spinal cords were hemisected so that the operated and control sides could be collected separately. The tissue was homogenized in homogenizing buffer (5 mM Tris, 2\% SDS, $2 \mathrm{mM}$ EDTA, $2 \mathrm{nM}$ EGTA, 2 mM phenylmethylsulfonylfluoride [PMFS], 10 $\mu \mathrm{M}$ Leupeptin Ph6.8). Samples were spun at 14,000 rpm for $10 \mathrm{~min}$, and the supernatant was collected. Protein concentration was determined using a Bio-Rad assay system (catalogue no. 500-0116) and a series of BSA standards. Samples were boiled for 5 min prior to SDS-polyacrylamide gel electrophoresis (PAGE). Equal amounts of $10 \mu \mathrm{g}$ of protein was loaded and separated at $200 \mathrm{~V}$ for $55 \mathrm{~min}$. Proteins were electrophoretically transferred to Hybond ${ }^{\mathrm{TM}}$ ECL $^{\mathrm{TM}}$ nitrocellulose membranes (Amersham Pharmacia Biotech). Membranes were blocked with
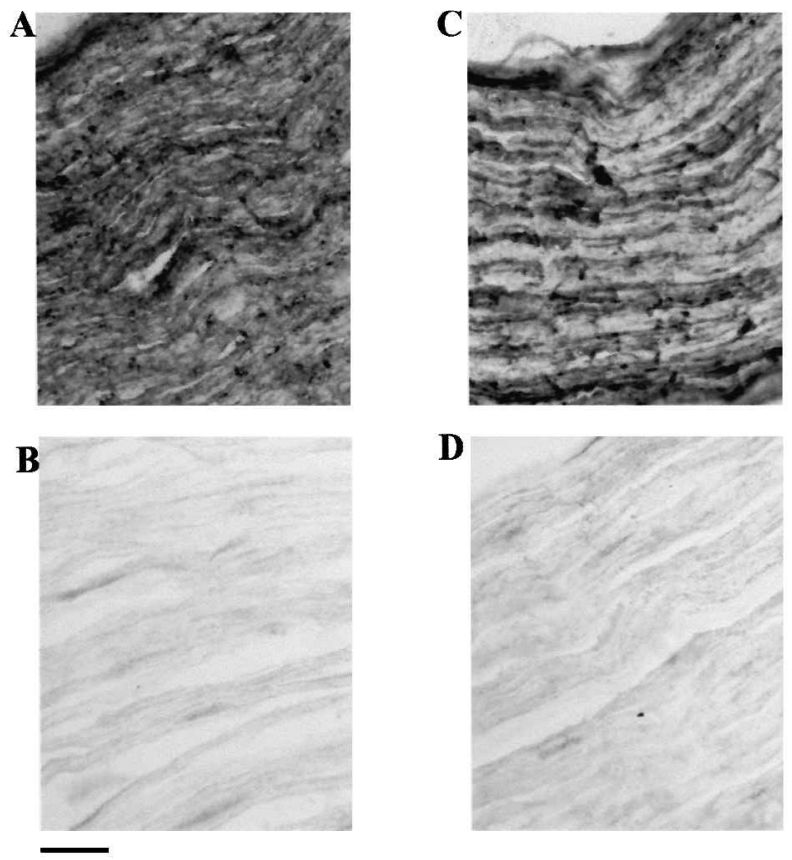

FIG. 3. Developmental pattern of hsp27 and hsp70 immunoreactivity in the sciatic nerve and the effect of neonatal nerve injury. The pattern of hsp27 (A) and hsp70 (B) immunoreactivity in normal sciatic nerves at P7 is shown. Hsp27 immunoreactivity is strong, and the staining for hsp27 remains at a high level throughout development. In contrast, hsp70 immunoreactivity is weak at all ages studied. The effect of sciatic nerve crush at birth on the expression of hsp27 (C) and hsp70 (D) at P7 is shown. It can be seen that neonatal nerve injury does not alter the pattern of staining for either of these proteins. Bar $=20 \mu \mathrm{m}$ 

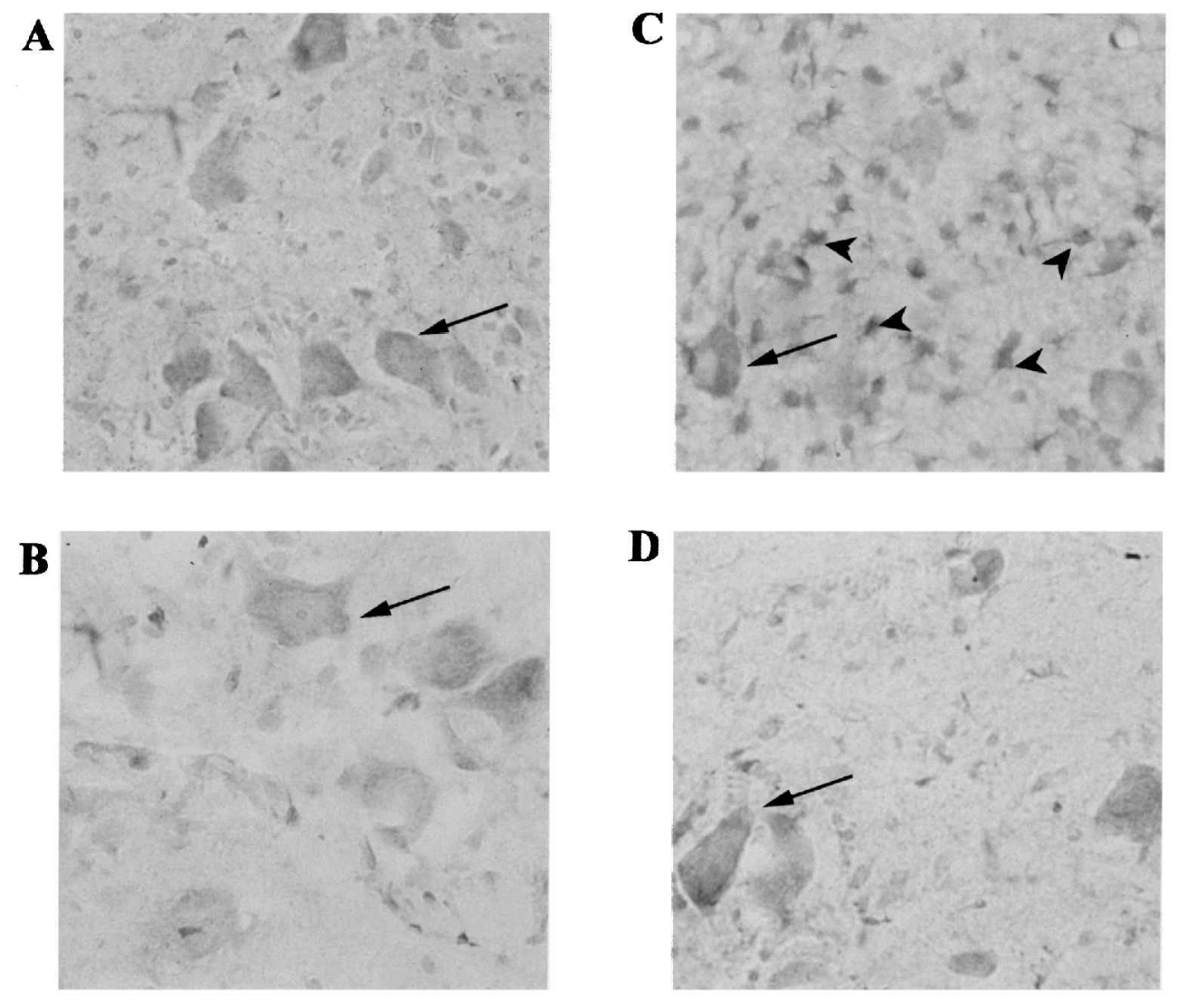

FIG. 4. Developmental pattern of hsp70 immunoreactivity in spinal cord motoneurones and the effect of neonatal nerve injury. Cross-sections of the ventral horn of the lumbar spinal cord stained for hsp70 are shown. The pattern of staining for hsp70 in motoneurones at birth is relatively weak and this staining intensity does not change with age, so that at P7 (A) and P21 (B) a similar pattern of immunoreactivity was observed. Furthermore, following sciatic nerve crush at birth, the expression of hsp70 is unaltered in motoneurones at P7 (C) and P21 (D) (arrows). However, there is a clear increase in the number of hsp70 positive cells (arrowheads) surrounding the injured motoneurones at P7 (C). Bar $=20 \mu \mathrm{m}$

$5 \%$ nonfat dried milk and $0.1 \%$ Triton X-100 in PBS for $1 \mathrm{~h}$ at room temperature. The blots were then incubated for $1 \mathrm{~h}$ at room temperature in the primary mouse monoclonal antibody to hsp70 at a dilution of 1:1,000 in PBS containing 5\% dried milk, 0.1\% Triton X-100 (Santa Cruz Biotechnology). After three washes in PBS containing $0.1 \%$ Triton X-100 for 15 min each, membranes were incubated in HRP conjugated anti-mouse (Dako catalog no. P0260) immunoglobulin at a dilution of 1:1,000 in PBS containing 5\% dried milk and $0.1 \%$ Triton X-100 for $1 \mathrm{~h}$. After three washes in PBS containing $0.1 \%$ Triton-X100 for $15 \mathrm{~min}$ each, membranes were treated with a chemiluminescent detection reagent (ECL ${ }^{\mathrm{TM}}$, Amersham Pharmacia Biotech, Arlington Heights, IL (Rochester, NY). Blots were visualized using Kodak film and fixation. Each Western blot contained samples from three animals in each group, and each sample was run on at least two blots. On each blot, equal protein quantities of brain homogenates were run next to the samples as standards.

The films were scanned using a KS300 image analysis program measuring pixel densities of each lines. Densities for the samples were normalized against densities of the brain standard in each blot. Results are presented as the mean and SEM of arbitrary units of line densities.

\section{Assessment of Apoptosis}

The presence of apoptotic motoneurones in the spinal cord of control and operated animals was assessed between P1 and P7, using either the TUNEL technique or staining for activated caspase-3 immunoreactivity.

TUNEL labeling. Spinal cord sections were stained with the Fluorescein In Situ Cell Death Detection Kit 
(Roche catalog no. 1684795 ) using the protocol provided. This method is based on terminal deoxynucleotidyl transferase (TdT)-catalyzed polymerization of fluorescently labeled nucleotides to free $3^{\prime}$-OH DNA end breaks occurring in apoptotic cell nuclei. Briefly, sections were permeabilized in $0.1 \%$ Triton $\mathrm{X}-100$ containing $0.1 \%$ sodium citrate for $2 \mathrm{~min}$ on ice. Following two rinses in PBS, the TUNEL reaction mixture was added to the sections and incubated at $37^{\circ} \mathrm{C}$ for $1 \mathrm{~h}$. The reaction mixture was removed by repeated washes in PBS. The sections were then examined under a fluorescent microscope using a blue filter for the detection of fluorescein-labeled apoptotic nuclei.

Caspase-3 immunohistochemistry. For activated caspase-3 immunoreactivity, the spinal cord sections were first blocked in $3 \%$ goat serum $(1 \mathrm{~h})$ and incubated overnight at $4{ }^{\circ} \mathrm{C}$ with the primary monoclonal antibody (PharMingen, catalog no. 559565, dilution 1:1500), specific for the activated form of the caspase-3 enzyme only. Negative control sections, in which the primary antibody was omitted, were also prepared. After thorough washing in PBS with $0.1 \%$ Triton-X-100 (Sigma), the sections were incubated for $2 \mathrm{~h}$ at room temperature in biotinylated secondary antibody (dilution 1:100; Vector). Texas Red Avidin D (Vector catalog no. A-2006, dilution 1:100) was used as a fluorescent layer. The presence of Texas Red-labeled hsp27 immunoreactivity assessed using a fluorescent microscope with a green filter.

Co-expression of hsp27 and apoptotic markers. In some experiments, the co-expression of hsp27 and markers of apoptosis in injured motoneurones was examined using either the TUNEL method or immunohistochemistry for activated caspase-3. For double-labeling experiments with TUNEL, the sections were first processed for TUNEL as described above and then immunostained for hsp27. In these experiments, Texas Red Avidin D (dilution 1:100) was used as a fluorescent layer for the hsp27 immunoreactivity, and the TUNEL-positive nuclei were labeled with fluorescein (as above).

For double-labeling experiments for hsp27 and caspase-3 immunoreactivity, following incubation in 3\% chicken serum for $1 \mathrm{~h}$, the sections were first stained for hsp27 using the primary antibody as described above. In these experiments, the secondary antibody was a fluorescein conjugate of a chicken anti-goat IgG (Chemicon catalog no. AP 163F, dilution 1:100). The sections were then processed for caspase-3, as described above.

In these double-labeling experiments, in order to quantify the number of hsp27 and/or caspase-3-positive motoneurones, we counted the number of motoneurones immunostained for each antibody in the sciatic pool, in five sections of each spinal cord. Each section was examined for the presence of motoneurones that were immunoreactive for both proteins, ie co-expressed hsp27 and caspase-3. Each experimental group consisted of four animals, and the effect of nerve injury at P0 on the immunoreactivity of these markers was assessed at three different time intervals: within the first $24 \mathrm{~h}$ (0-1 days), and 4 and 7 days after the injury. The sections were examined under a fluorescent microscope and the presence of hsp27 assessed using a green filter for fluorescein, and caspase-3-positive cells counted using a blue filter for detection of Texas Red. The mean number of motoneurones reactive for each protein was calculated for each section and the data is presented as the mean with the standard error of mean (SEM).

\section{Statistical Analysis}

The results were analyzed using the Mann-Whitney $U$ test for comparison of independent samples. Two-tailed tests were used in all instances, and significance was set at $p<0.05$.

\section{RESULTS}

\section{Pattern of Immunoreactivity for hsp27 \\ and hsp70 in Motoneurones and Sciatic \\ Nerve during Postnatal Development}

Hsp27 immunoreactivity. (1) Hsp27 immunoreactivity in developing motoneurones: Immunostaining for hsp27 was carried out on sections from the lumbosacral segment of the spinal cord of rats at various stages during postnatal development from P0 to P28. Figure 1 shows examples of spinal cord sections stained for hsp27 immunoreactivity and illustrates the pattern of hsp27 immunoreactivity observed within motoneurones at different developmental stages. As can be seen in Figure 1, the intensity of staining for hsp27 within motoneurone cell bodies increases with development. The distribution of hsp27 protein was even within the cytoplasm, soma and perikaryon region, although no nuclear staining for hsp27 was observed. The number of hsp27 immunoreactive motoneurones also increased with age. The number of hsp27 motoneurones was counted in each spinal cord at each developmental stage and the results are summarized in Figure 2. At birth, some motoneurones are already positive for hsp27 (Fig. 1A) and in rats aged between P0 and $\mathrm{P} 4,63 \%( \pm 2.6 \% \mathrm{SEM} ; n=6)$ of motoneurones are hsp27 immunoreactive (Fig. 2). With development, the proportion of hsp27 expressing motoneurones gradually increases, so that between P7 and P14, 67\% $( \pm 3.0 \%$ $\mathrm{SEM} ; n=4$ ) of sciatic motoneurones are hsp27 positive. 

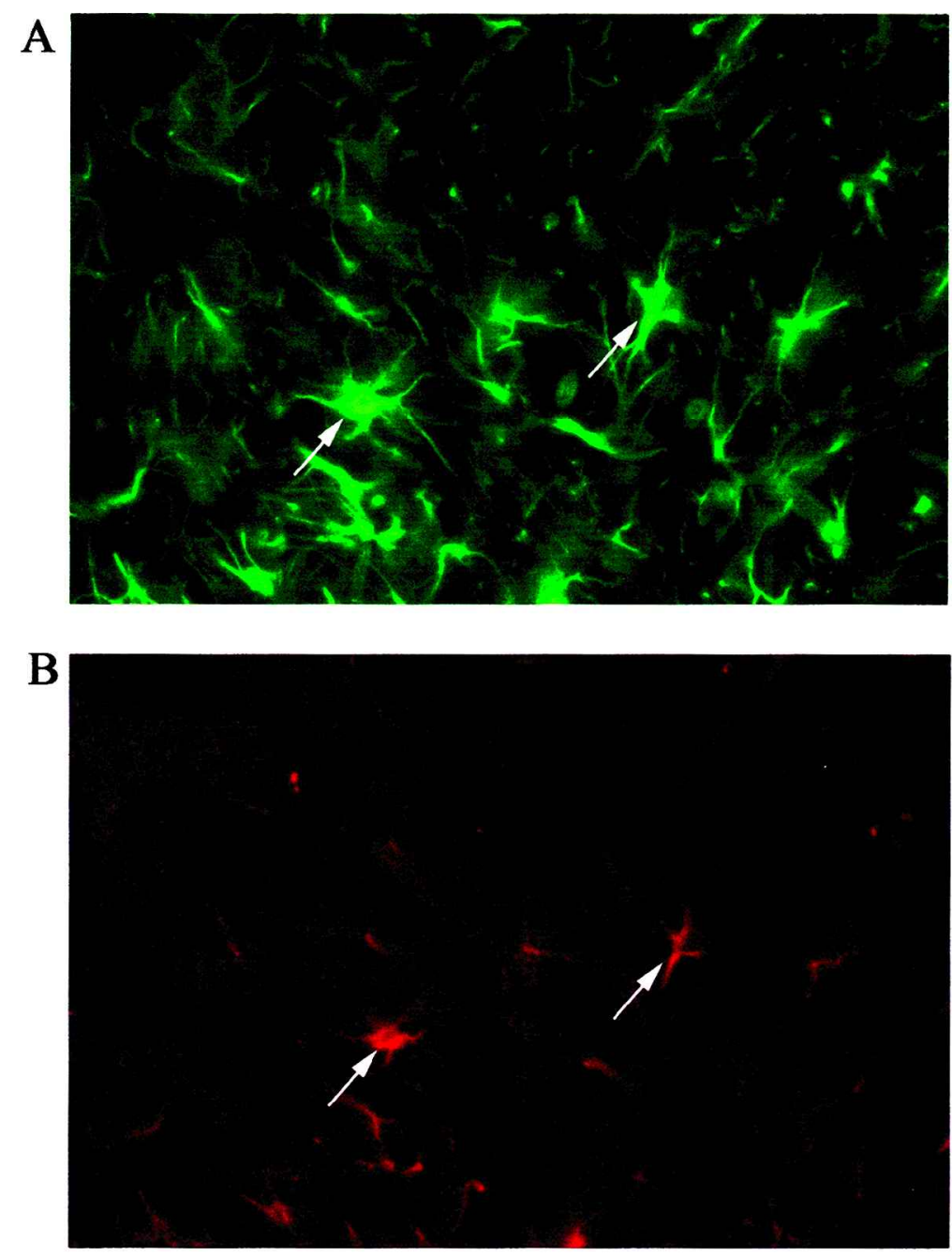

FIG. 5. Expression of hsp70 in GFAP-positive astroglia following neonatal nerve injury. Seven days after sciatic nerve crush at birth, spinal cord sections were double-stained for GFAP and hsp70 7 days after injury using immunofluorescence. (A) An example of the pattern of GFAP immunoreactivity in astroglia in the ventral horn of the operated side of the spinal cord. The same section stained for hsp70 is shown in B). (B) It can be seen that the hsp70 positive cells (arrows) are also immunoreactive for GFAP (arrows) and are therefore likely to be astroglia. Bar $=50-\mu \mathrm{m}$.

By day 21, a dramatic increase in the number of hsp27 immunoreactive motoneurones occurs, so that almost all sciatic motoneurones express the protein (Figs. 1D and 2; $95 \% \pm 1.1 \%$ SEM, $n=4 ; p<0.01)$. (2) Hsp $27 \mathrm{im}$ munoreactivity in the sciatic nerve: The sciatic nerves from animals aged between P0 and P28 were processed for hsp27 immunoreactivity, and an example is shown in Figure 3A. Strong specific immunoreactivity for hsp27 was detected in the sciatic nerve at all ages examined, although the precise location of this staining, either within the axons or in Schwann cells, is not obvious under the light microscope. The intensity of staining was evenly distributed along the length of the nerve examined, with no proximal-distal differences apparent and did not change significantly with age.

Hsp70 immunoreactivity. (1) Hsp70 immunoreactivity in developing motoneurones: The developmental pattern of hsp70 immunoreactivity within sciatic motoneurones in rats aged between $\mathrm{P} 0$ and $\mathrm{P} 28$ was also examined. At P0, hsp70 immunoreactivity in motoneurones is very low and the intensity of staining does not increase significantly with development. Examples of spinal cord sections stained for hsp70 at 7 and 21 days 
A

\section{Control Operated}

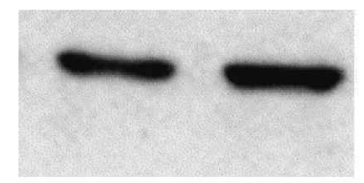

B

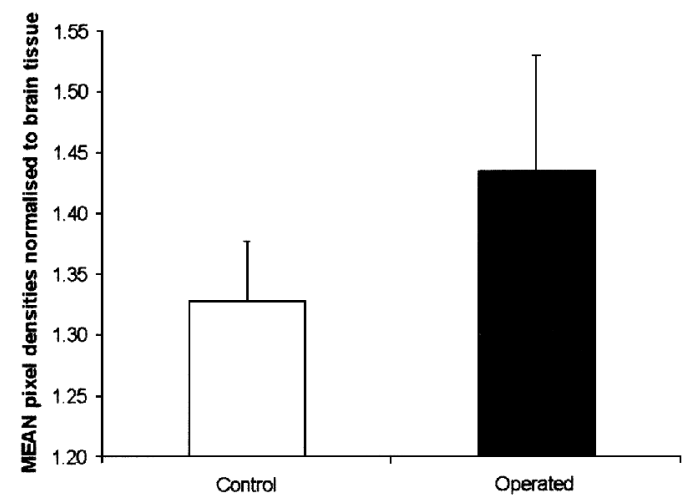

FIG. 6. Hsp70 levels in spinal cords following neonatal nerve injury. Western blot analysis of hsp70 levels in spinal cord samples from control and operated animals were processed 7 days following sciatic nerve crush at birth. (A) An example of such a blot where several spinal cords were pooled $(n=3)$. Eight Western blots were run from both operated and control animals and the mean band density of each blot was measured. (B) The results are summarized. There was a slight but nonsignificant increase in the mean band density of the operated samples.

are shown in Figure 4A and 4B, respectively. At each age, the staining for hsp70 was weak but specific in all motoneurones within the lumbosacral region. This lowintensity hsp70 immunoreactivity was not present in the negative control sections in which the primary antibody was omitted. The cellular distribution of hsp70 was rather characteristic for the cell bodies and towards the perikarya the intensity of staining decreased. (2) Hsp70 immunoreactivity in the sciatic nerve: The developmental immunoreactivity of hsp70 was also examined in sections of the sciatic nerve. At all ages studied, hsp70 immunoreactivity was very weak along the length of the dissected sciatic nerve (Fig. 3B). The intensity and pattern of hsp70 staining does not change with distance from the cell body or with age.
Effect of Neonatal Nerve Injury on hsp27 and hsp70 Immunoreactivity in Motoneurones and Sciatic Nerve

The effect of sciatic nerve crush at birth on the developmental pattern of hsp27 and hsp70 immunoreactivity within the spinal cord and the sciatic nerve was examined at various developmental stages.

Hsp27 immunoreactivity. (1) Hsp27 immunoreactivity in motoneurones following neonatal nerve injury: Following sciatic nerve crush at birth, the immunoreactivity of hsp27 in motoneurones was examined at intervals between P0 and P28. Examples of the pattern of staining observed are shown in Figure 1E-H. The number of motoneurones positive for hsp27 was counted at each age, and the pattern of immunoreactivity assessed. Within the first few days of injury, hsp27 immunoreactivity in injured motoneurones is similar to that observed in control, unoperated motoneurones at the same age. However, by 4 days after injury, the intensity of staining for hsp27 is significantly greater than that observed in control motoneurones (Fig. 1E) and peaks at 7 days (Fig. 1F). Not only is the staining for hsp27 in motoneurones more intense after injury, but the proportion of motoneurones that are hsp27 immunoreactive at each age is greater following nerve injury than normal, although there are fewer surviving motoneurones present following the injury. Thus, the results summarized in Figure 2 show that in control, unoperated animals aged between P0 and P4, $63 \%( \pm 2.6 \% \mathrm{SEM} ; n=6)$ of sciatic motoneurones express hsp27, whereas following nerve injury $75 \%$ $( \pm 2.1 \% \mathrm{SEM} ; n=4)$ of those motoneurones that survive express hsp27 at this stage. This difference in the immunoreactivity of hsp27 in operated and unoperated motoneurones is significant $(p<0.01)$ and is also apparent at later stages (Fig. 2). Between P7 and P14, 83.6\% $( \pm 2.8 \% \mathrm{SEM} ; n=6)$ of sciatic motoneurones in the operated side of the spinal cord are immunoreactive for hsp27, compared to only 67\% ( $\pm 3.0 \%$ SEM; $n=4 ; p<$ 0.02 ) of uninjured motoneurones. By 21 days, the intensity of hsp 27 immunoreactivity in the injured motoneurones is similar to that in control uninjured motoneurones, and has reached adult levels (Fig. 1D,H). In addition, by $\mathrm{P} 21$, there is no significant difference in the proportion of motoneurones that express hsp27 in injured and uninjured animals and almost all sciatic motoneurones are hsp27 positive (Figure 2). (2) Hsp27 immunoreactivity in the sciatic nerve following neonatal nerve injury: The effect of nerve injury on hsp27 immunoreactivity within the sciatic nerve was also examined. Nerve crush at birth did not alter the immunoreactivity for hsp27, which was 

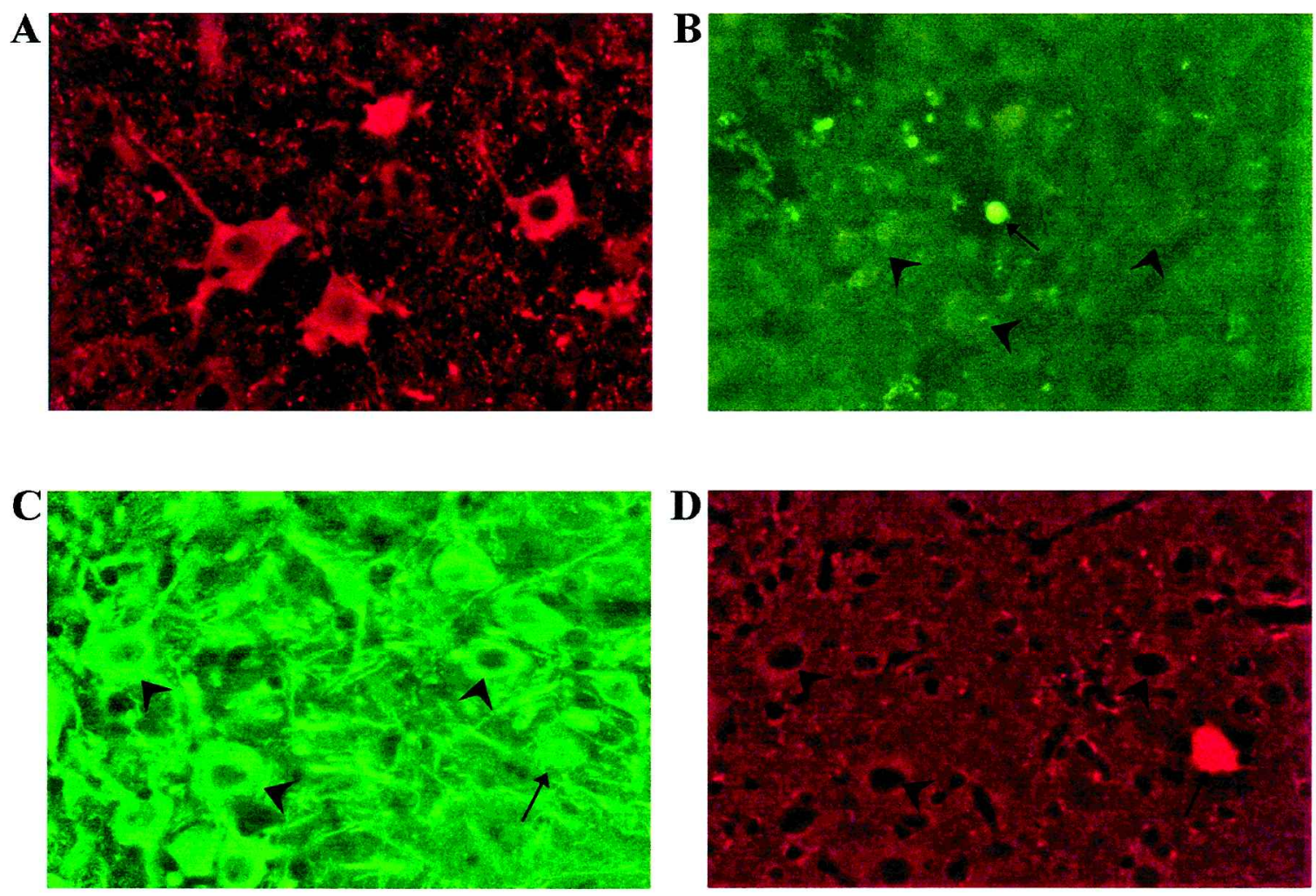

FIG. 7. Apoptosis and hsp27 expression in motoneurones following neonatal nerve injury. Cross-sections of the operated ventral horn from a P4 animal are shown following sciatic nerve crush at birth. Sections were processed for double labeling for hsp27 immunoreactivity as well as markers of apoptosis. (A) hsp27 immunoreactivity labeled with Texas Red can be seen within several sciatic motoneurones (arrowheads), although some motoneurones do not express hsp27 (arrow). (B) The same section, stained for TUNEL and labeled with fluorescein is shown using the same field of view. It can be seen that the TUNEL positive motoneurone (arrow) is not hsp27 immunoreactive. No hsp27-positive motoneurones stained for TUNEL (arrowheads). A different section stained for hsp27 (C) and caspase-3 (D) immunoreactivity is shown. Many hsp27 immunoreactive motoneurones, labeled with fluorescein can be seen (arrowheads C). The same section and field of view shows double labeling for caspase-3 immunoreactivity using Texas Red (D). A single motoneurone strongly immunoreactive for caspase-3 (arrow) can be seen, but no hsp27 positive motoneurones co-express this marker of apoptosis (arrowheads). Bar $=20 \mu \mathrm{m}$

similar to that observed in normal control sciatic nerves (Fig. 3A,C). The pattern and intensity of staining at all time points examined, was not altered by nerve injury and was similar along the entire length of the nerve examined, including the site of injury as well as the proximal and distal segments.

Hsp70 immunoreactivity. (1) Hsp70 immunoreactivity in motoneurones following neonatal nerve injury: Following nerve injury, the pattern of hsp70 immunoreactivity in injured motoneurones was examined at various intervals between P0 and P28. Examples of the pattern of staining observed in the operated ventral horns at P7 and P21 are shown in Figure 4C,D. Following nerve injury at birth there was no change in the intensity of hsp70 immunoreactivity within motoneurones in the sciatic mo- tor pool, at any stage examined. However, there are clearly fewer motoneurones present in the operated ventral horn compared to normal, as, by P7, more than 50\% of motoneurones have already died (Lawson and Lowrie, 1998). In addition, although a few hsp70 immunoreactive glial cells were observed in normal ventral horns, following nerve injury there was a dramatic astrogliosis around the injured motoneurones and many of these glial cells were hsp70 immunoreactive. Double-labeling immunofluorescence staining using an antibody to GFAP, a specific marker for astroglia, revealed that the hsp70 immunoreactive glia were predominantly astrocytes (Fig. 5). In order to see whether there were any changes in the overall levels of hsp70 within the spinal cord following nerve injury, western blot analysis of hsp70 was carried out on normal and injured spinal cords 7 days after in- 


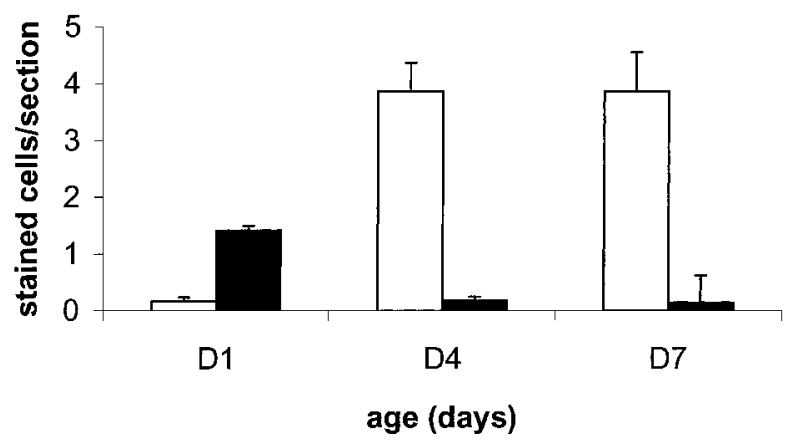

FIG. 8. The number of hsp27 and Caspase-3 positive motoneurones in the injured sciatic motor pool. The number of hsp 27 and caspase- 3 positive motoneurones was established in the operated ventral horn 1, 4, and 7 days after nerve injury at birth. Each group consisted of four animals, and five sections were examined from each spinal cord. The results are presented as the mean number of stained motoneurones per section, and the error bars show the standard error of mean. The empty bars show the number of hsp27-positive motoneurones, and the filled bars show the caspase-3-positive motoneurones. It can be seen that the proportion of motoneurones immunoreactive for caspase- 3 is greatest 1 day after injury and decreases rapidly thereafter. In contrast, few hsp27-positive motoneurones are present at $\mathrm{P} 1$, and the number of immunoreactive motoneurones increases dramatically by $\mathrm{P} 4$.

jury. An example of such a blot is shown in Figure 6A. The mean pixel density from eight different blots is shown in the bar diagram (Fig. 6B), which illustrates that there is no significant change in hsp70 levels in spinal cords following nerve injury. However, significant motoneurone death has already occurred in the injured spinal cord. Since normal, uninjured motoneurones are hsp70 positive, it appears that other cell types, including astroglia, may be contributing to the levels of hsp70 observed in the operated tissue. (2) Hsp70 immunoreactivity in the sciatic nerve: The effect of neonatal nerve injury on hsp70 immunoreactivity within the sciatic nerve was also examined. Examples of the pattern of staining found are shown in Figure 3D. Nerve injury had no effect on the pattern of hsp70 immunoreactivity at any ages studied and was similar along the entire length of the nerve, including the site of injury as well as the proximal and distal segments of the nerve.

Co-expression of hsp27 and markers of apoptosis in motoneurones following neonatal nerve injury. The results showed that the intensity of staining for hsp27 was increased in motoneurones following nerve injury. Since many motoneurones are known to die by apoptosis following neonatal nerve injury, we next examined whether hsp27-positive motoneurones were also positive for markers of apoptosis. One, 4 and 7 days after neonatal nerve injury, spinal cord sections from at least three animals at each age were processed for double labelling of hsp27 and markers of apoptosis as described below. (1) TUNEL labeling of injured motoneurones: Spinal cord sections were processed for TUNEL and subsequently immunostained for hsp27. An example of a section stained for both TUNEL and hsp27 is shown in Figure 7A,B. At all ages studied, no motoneurones in the contralateral control ventral horn were found to be TUNEL positive. In the operated ventral horn, very few motoneurones were found to be TUNEL positive. However, as early as 1 day after injury, some TUNEL-positive cells could be seen in the operated ventral horn, and TUNEL staining could still be detected at P7. Although some motoneurones expressed hsp27 within 1 day of injury, there was no increase in the intensity of hsp27 immunoreactivity prior to $\mathrm{P} 4$. In all sections examined, at all stages, no hsp27 immunoreactive motoneurones were also found to be positive for TUNEL. (2) Caspase-3 immunoreactive motoneurones: Spinal cord sections were processed for hsp27 immunoreactivity and subsequently for caspase- 3 reactivity. An example of a section stained for both hsp27 and caspase-3 immunoreactivity is shown in Figure 7C,D. Capase-3-immunoreactive motoneurones were found in the operated ventral horn in each section, although no positive cells were present at any stage in the control ventral horn. The number of caspase-3-immunoreactive motoneurones in the operated ventral horn per section was established. As can be seen in Figure 8, the number of caspase-3-positive motoneurones was greatest 1 day after nerve injury and declined rapidly by 7 days. Thus, there were $1.66( \pm 0.08$ SEM) caspase3 -positive motoneurones per section at $\mathrm{P} 1$, which had declined to 0.20 ( \pm 0.07$)$ motoneurones per section by $\mathrm{P} 4$, and by $\mathrm{P} 7$ only 0.18 ( \pm 0.15 SEM) motoneurones per section were stained for caspase-3. In contrast, hsp27 immunoreactivity was low 1 day after injury and increased dramatically by 4 days. There were 0.16 ( \pm 0.06 SEM) hsp27-immunoreactive motoneurones per section at P1, 3.90 ( \pm 0.5 SEM) motoneurones at P4, and by P7, 3.95 ( $\pm 0.69 \mathrm{SEM}$ ) hsp27-positive motoneurones. At no stage were any hsp27-positive motoneurones found to also be immunoreactive for caspase-3, although hsp27-positive motoneurones could be found in close proximity to caspase-3-positive motoneurones (Fig. 7C,D).

\section{DISCUSSION}

In this study, we investigated the expression of hsp27 and hsp70 proteins in developing rat spinal cord motoneurones within the sciatic motor pool, as well as their 
expression in peripheral sciatic nerves. In addition, we examined the effect of neonatal nerve crush on hsp27 and hsp70 immunoreactivity in injured motoneurones and axons. The results of this study show that hsp70 is constitutively expressed in motoneurones and axons throughout development, although at relatively low levels. Following neonatal nerve injury, the pattern and intensity of staining for hsp70 did not change in motoneurones or axons. However, there was a clear increase in the number of glial cells which stained for hsp70 within the injured ventral horn. It is well established that following neonatal nerve injury an extensive astrogliosis takes place in the spinal cord around the injured motor pool (Gilmore et al., 1990). In this study, there was a marked astrogliosis in the operated ventral horn, with a dramatic increase in the number of GFAP positive astrocytes around the injured motor pool. Double immunofluorescence revealed that those nonneural cells which were hsp70 immunoreactive were also positive for GFAP, and were therefore likely to be astrocytes.

Using Western blot analysis, it was not possible to detect any significant changes in hsp70 expression levels following neonatal nerve injury. The results showed that there was no difference in the mean pixel density of spinal cord samples taken from normal and operated rats, 7 days after neonatal nerve injury. However, by this stage, it is likely that many motoneurones will have already died as a consequence of the nerve injury (Lawson and Lowrie, 1998). Since we have established that motoneurones constitutively express hsp70, it is surprising that levels of hsp70 in the spinal cords from operated animals are as high as that found in normal animals. However, it is likely that an increase in the number of hsp70 positive astrocytes following injury may maintain overall levels of hsp70 within the injured spinal cord.

This study also examined the expression of hsp27. Hsp27 has been shown to be constitutively expressed in both sensory and motor neurones (Plumier et al., 1997). In mammalian cells hsp27 is thought to play a role in cytoskeletal microfilament organization (Lavoie et al., 1993). Therefore, it is likely that it plays an important role in normal cell body, as well as axon and dentrite structure. This may explain its relatively high level of expression in sciatic nerves at all ages studied here, although it is possible that the staining for hsp27 may be attributed to Schwann cell immunoreactivity. The results of this study show that in motoneurone cell bodies, hsp27 is developmentally regulated, so that the number of immunoreactive motoneurones as well as intensity of staining increases with age. Following nerve injury, hsp27 immunoreactivity increases within motoneurones, but only 4 days after injury. Furthermore, a greater proportion of motoneurones were hsp27 immunoreactive than in nor- mal spinal cords of the same age. Whether this indicates that those motoneurones that do not express hsp27 are more likely to die after injury, or that the injury itself induces hsp27 expression in more motoneurones, is not clear from these results. It is well established that the more immature a motoneurone is the more vulnerable it is to nerve injury (Greensmith and Vrbova, 1996). Following neonatal axotomy, $80 \%$ of sciatic motoneurones have died within the first two weeks of injury (Schmalbruch, 1984). In contrast, nerve injury in the adult results in no motoneurone death. Since hsp27 expression and intensity increases in motoneurones during development, we favour the possibility that those motoneurones that express hsp27 are more resistant to nerve injury. Indeed, in the adult, where nerve injury does not result in any motoneurone death, all motoneurones are strongly hsp27 positive. Thus, it is possible that among other factors involved in the resistance of adult motoneurones to nerve injury, the high expression of hsp27 in adult motoneurones may help them to maintain their normal function following an insult to the nerve.

Several lines of evidence that indicate that both developing neurones and glial cells die by apoptosis following injury to a peripheral nerve or to the spinal cord (Lawson and Lowrie, 1998; Liu et al., 1997; Oliviera et al., 1997), although some necrotic cell death does take place ( $\mathrm{Li}$ et al., 1998). In this study, we found that hsp27 immunoreactive motoneurones did not co-express markers of apoptosis. However, hsp27 immunoreactivity was only upregulated 4 days after injury, when many motoneurones had already died, most probably by apoptosis (Lawson and Lowrie, 1998). Although a few apoptotic motoneurones were found at P4 and P7, these neurones did not stain for hsp27. Therefore it is possible that those motoneurones that express hsp27 at high levels are less likely to undergo apoptosis and are therefore more capable of surviving nerve injury (Lewis et al., 1999). However, apoptotic markers such as TUNEL and caspase-3 label cells only within in a certain period of cell death. These markers therefore will not detect all apoptotic cells at all stages of apoptosis, particularly in vivo. Thus, we cannot exclude the possibility that some hsp27 positive motoneurones are undergoing apoptosis. Indeed, considering the high proportion of motoneurones that upregulate hsp27 expression at $\mathrm{P} 4$ and $\mathrm{P} 7$, and the fact that a large proportion of motoneurones will eventually die following nerve injury at birth, it is likely that even those motoneurones that upregulate their hsp27 expression are susceptible to cell death, either because they are unable to increase their hsp27 expression to a high enough level or they do so too late to prevent themselves from entering a cell death pathway. Therefore following neonatal nerve injury, hsp27 may be beneficial only to those motoneurones that are 
able to switch on this protective protein synthesis before the apoptotic cascade begins.

Interestingly, there are other lines of evidence that also indicate that hsps may play a role in protecting cells against apoptosis. In vitro, a variety of cells that over-express hsp27 or hsp70 have been shown to be more resistant to cell death (Sakurai et al., 1998; Uney et al., 1994; Wagstaff et al., 1999). In addition, using double labeling for apoptosis (TUNEL), both in vitro (Kobayashi et al., 2000) and in vivo (Lewis et al., 1999) studies have shown that cells expressing hsps at high levels do not undergo apoptosis. The mechanism by which hsp expression may prevent apoptosis has been also investigated. Bruey (2000) found that hsp27 can bind to cytochrome c released from mitochondria and can thereby inhibit the initial step in the apoptotic cascade, namely the interaction of Apaf1 with procaspase-9. A similar mechanism of action has been suggested by Beere (2000) for hsp70, whereby hsp70 binds to Apaf-1. These findings, taken together with the results of the present in vivo study suggest that the increased hsp27 expression in injured motoneurones and hsp70 in astrocytes may play a protective role in motoneurone survival following neonatal nerve injury.

\section{ACKNOWLEDGMENTS}

We would like to thank Dr. J. Dekkers for her help with the surgical procedures and Mr. J. Dick for his excellent technical assistance. Bernadett Kalmar is a research fellow funded by BIOREX Research and Development Ltd., Hungary. We are grateful to Biorex for their support. Linda Greensmith is The Graham Watts Senior Research Fellow, funded by the Brain Research Trust.

\section{REFERENCES}

AMIN, V., CUMMING, D.V. and LATCHMAN, D.S. (1996). Over-expression of heat shock protein 70 protects neuronal cells against both thermal and ischaemic stress but with different efficiencies. Neurosci. Lett. 206, 45-48.

BARBE, M.F., TYTELL, M., GOWER, D.J., et al. (1988). Hyperthermia protects against light damage to the retina. Science 241, 1817-1820.

BECKER, J. and CRAIG, E.A. (1994). Heat-shock proteins as molecular chaperones. Eur. J. Biochem. 219, 11-23.

BEERE, H.M., WOLF, B.B., CAIN, K., et al. (2000). Hsp 70 inhibits apoptosis by preventing recruitment of procaspase9 to the Apaf-1 apoptosome. Nat. Cell Biol. 2, 469-475.

BILBAO, F.D., GIANNAKOPOULOS, P., SRINIVASAN, A., et al. (2000). In vivo study of motoneuron death induced by nerve injury in mice deficient in the caspase 1/interleukin-1Bconverting enzyme. Neuroscience 98, 573-583.

BROWN, I.R., RUSH, S.J. and IVY, G.O. (1989). Induction of heat shock gene at the site of tissue injury in the rat brain. Neuron 2, 1559-1564.

BRUEY, J.M., DUCASSE, C., BONNIAUD, P., et al. (2000). Hsp27 negatively regulates cell death by interacting with cytochrome c. Nat. Cell Biol. 2, 645-652.

COGGESHALl, R.E., LEKAN, H., DOUBELL, T.P., et al. (1997). Central changes in primary afferent fibers following peripheral nerve lesions. Neuroscience 77, 1115-1122.

COHEN, M.V. and DOWNEY, J.M. (1983). Ischaemic preconditioning: can the protection be bottled? Lancet 342, 6 .

COSTIGAN, M., MANNION, R.J., KENDALL, G., et al. (1998). Heat shock protein 27: developmental regulation and expression after peripheral nerve injury. J. Neurosci. 18, 5891-5900.

GERNOLD, M., KNAUF, U., GAESTEL, M., et al. (1993). Development and tissue-specific distribution of mouse small heat shock protein hsp25. Dev. Gen. 14, 103-111.

GILMORE, S.A., SIMS, T.J. and LEITING, J.E. (1990). Astrocitic reactions in spinal gray matter following sciatic axotomy. Glia 3, 342-349.

GREENSMITH, L. and VRBOVÁ, G. (1996). Motoneurone survival: a functional approach. Trends Neurosci. 19, 450-455.

HEIKKILA J.J. (1993). Heat shock gene expression and development II. An overview of mammalian and avian developmental systems. Dev. Genet. 14, 87-93.

HOPKINS, D.A., PLUMIER, J.L. and CURRIE, R.W. (1998). Induction of the $27-\mathrm{kDa}$ heat shock protein (Hsp27) in the rat medulla oblongata after vagus nerve injury. Exp. Neurol. 153, 173-183.

HOUENOU, L.J., LI, L., LEI, M., et al. (1996). Exogenous heat shock cognate protein Hsc 70 prevents axotomy-induced death of spinal sensory neurones. Cell Stress Chaperones 1, 161-166.

KOBAYASHI, Y., KUME, A., LI, M., et al. (2000). Chaperones Hsp70 and Hsp40 suppress aggregate formation and apoptosis in cultured neuronal cells expressing trunkated androgen receptor protein with expanded polyglutamine tract. J. Biol. Chem. 275, 8772-8778.

LAVOIE, J.G., GINGRAS-BRETON, G., TANGUAY, R.M., et al. (1993). Induction of chinese hamster hsp27 gene expression in mouse cells confers tolerance to heat shock. Hsp27 stabilization of the microfilament organization. J. Biol. Chem. 268, 3420-3429.

LAWSON, S.J. and LOWRIE, M.B. (1998). The role of apoptosis and excitotoxicity in the death of spinal motoneurons and interneurons after neonatal nerve injury. Neuroscience 87, 337-348.

LEWIS, S.E., MANNION, R.J., WHITE, F.A., et al. (1999). A 
role of hsp27 in sensory neuron survival. J. Neurosci. 19, 8945-8953.

LI, L., HOUENOU, L.J., WU, W., et al. (1998). Characterization of spinal motoneuron degeneration following different types of peripheral nerve injury in neonatal and adult mice. J. Comp. Neurol. 396, 158-168.

LI, Y., CHOPP, M., ZHANG, Z.G., et al. (1993). Neuronal survival is associated with $72-\mathrm{kDa}$ heat shock protein expression after transient middle cerebral artery occlusion in the rat. J. Neurol. Sci. 120, 187-194.

LIU, X.Z., XU, X.M., HU, R., et al. (1997). Neuronal and glial apoptosis after traumatic spinal cord injury. J. Neurosci. 17, 5395-5406.

LOWRIE, M.B., KRISHNAN, S. and VRBOVÁ, G. (1982). Recovery of slow and fast muscles following nerve injury during early post-natal development in the rat. J. Physiol. (Lond.) 331, 51-66.

MANZERRA, P., and BROWN, I.R. (1992). Expression of heat shock genes Hsp70 in the rabbit spinal cord: localization of constitutive and hyperthermia-inducible mRNA species. J. Neurosci. Res. 31, 606-615.

MAYER, J., and BROWN, I. (eds) (1994). Heat Shock Proteins in the Nervous System. Academic Press: London.

MORENO-FLORES, M.T., OLAZABAL, U.E. and KREUTZBERG, G.W. (1997). Axotomy increases the expression of glucose-regulated protein $78 \mathrm{kDa}$ in rat facial nucleus. Exp. Neurol. 146, 10-16.

MOSELEY, P.L. (1997). Heat shock proteins and heat shock adaptation of the whole organism. J. Appl. Physiol. 83, 14131417.

NEW, G.A., HENDRICKSON, B.R. and JONES, K.J. (1989). Induction of heat shock protein $70 \mathrm{mRNA}$ in adult hamster facial nuclear groups following axotomy of the facial nerve. Metab. Brain Dis. 4, 273-479.

OLIVIERA, A.L., RISLING, M., DECKNER, M., et al. (1997). Neonatal sciatic nerve transection induces TUNEL labeling of neurons in the rat spinal cord and DRG. Neuroreport 8, 2837-2840.

PLUMIER, J.C.L., HOPKINS, D.A., ROBERTSON, H.A., et al. (1997). Constitutive expression of the $27-\mathrm{kDa}$ heat shock protein (Hsp27) in sensory and motor neurons of the rat nervous system. J. Comp. Neurol. 384, 409-428.

ROMANES, G.J. (1946). Motor localisation and the effects of nerve injury on the ventral horn cells of the spinal cord. J. Anat. 80, 11-131.
SAKURAI, M., HAYASHI, T., ABE, K., et al. (1998). Enhancement of heat shock protein expression after transient ischaemia in the preconditioned spinal cords of rabbits. J. Vasc. Surg. 27, 720-725.

SAMALI, A., and COTTER, T.G. (1996). Heat shock proteins increase resistance to apoptosis. Exp. Cell Res. 223, 163170.

SCHLESINGER, M.J. (1985). Stress response in avian cells, in: Changes in Eukaryotic Gene Expression in Response to Environmental Stress. B.G. Atkinson and D.B. Walden (eds), Academic Press, Inc.: Orlando, FL, pps. 183-196.

SCHMALBRUCH, H. (1984). Motoneurone death after sciatic nerve section in newborn rats. J. Comp. Neurol. 224, 252258.

SPRINGER, J.E., AZBILL, R.D., and KNAPP, P.E. (2000). Activation of caspase- 3 apoptotic cascade in traumatic spinal cord injury. Nat. Med. 5, 943-946.

UNEY, J.B., STALEY, K., TYERS, P., et al. (1994). Transfection with hsp70i protects rat dorsal root ganglia neurons and glia from heat stress. Gene. Ther. Suppl 1, 65.

VANDERLUIT, J.L., McPHAIL, L.T., FERNANDES, K.J., et al. (2000). Caspase-3 is activated following axotomy of neonatal facial neurons and caspase- 3 gene deletion delays axotomy-induced cell death in rodents. Eur. J. Neurosci. 12, 3469-3480.

WAGSTAFF, M.J.D., COLLACO-MORAES, Y., SMITH, J., et al. (1999). Protection of neural cells from apoptosis by Hsp27 delivered with a Herpes symplex virus-based vector. J. Biol. Chem. 274, 5061-5069.

XIA, H., IKATA, T., KATOH, S., et al. (1998). Whole body hyperthermia selectively induces heat shock protein 72 in neurons of the rat spinal cord. Neurosci. Lett. 258, 151154.

YENARI, M.A., FINK, S.L., SUN, G.H., et al. (1998). Gene therapy with Hsp72 is neuroprotective in rat models of stroke and epilepsy. Ann. Neurol. 44, 584-591.

Address reprint requests to: Linda Greensmith, Ph.D. Sobell Department of Neurophysiology Institute of Neurology Queen Square London WCIN 3BG, U.K.

E-mail: 1.greensmith@ion.ucl.ac.uk 